\title{
Impactos da Volatilidade da Taxa de Câmbio no Comércio Setorial do Mercosul ${ }^{\dagger}$
}

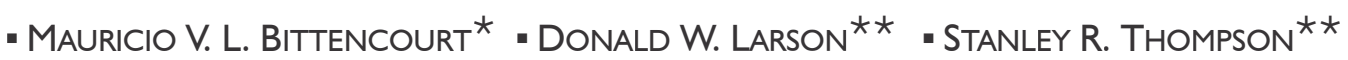

\begin{abstract}
RESUMO
Este estudo capta o impacto da volatilidade da taxa de câmbio real bilateral no comércio. Estima-se um modelo gravitacional setorial utilizando-se duas medidas de volatilidade da taxa de câmbio. Os resultados mostram que uma redução na volatilidade da taxa de câmbio, o crescimento da renda e a redução das tarifas comerciais contribuem para aumentar o comércio bilateral no Mercosul. Este estudo sugere $a$ inclusão e implementação de políticas comuns, estáveis e integradas entre os países do Mercosul, de modo a reduzir os impactos adversos da volatilidade cambial no comércio dos países envolvidos.
\end{abstract}

\section{Palavras-chave}

comércio bilateral, volatilidade da taxa de câmbio, econometria de painel, modelos gravitacionais, mercosul

\begin{abstract}
This study captures the impact of real bilateral exchange rate volatility on trade. A sectoral gravity model is estimated under two different measures of exchange rate volatility. Results show that a reduction in exchange rate volatility, an increase in the country's income, and a reduction in trade tariffs can increase bilateral trade in Mercosur. This study suggests the inclusion and implementation of common, stable, and integrated policies among Mercosur countries, aiming to reduce the adverse effects of the exchange rate volatility on bilateral trade among these countries.
\end{abstract}

KEY WORDS

bilateral trade, exchange rate volatility, panel econometrics, gravity models, Mercosur

\section{JEL Classification}

FI3, FI5, E6I

- Os autores gostariam de agradecer o suporte financeiro do CNPq, e o apoio de Samuel Munyaneza e da Conferência de Comércio e Desenvolvimento das Nações Unidas (UNCTAD) por gentilmente terem disponibilizado os dados utilizados neste estudo.

* Professor do Departamento de Economia da Universidade Federal do Paraná (UFPR). E-mail: mbittencourt@ufpr. br. End. para contato: UFPR/PPGDE - Av. Pref. Lothário Meissner , 3400 - Campus III - Jardim Botânico - 802I0170 - Curitiba- Paraná.

* Professores do Department of Agricultural, Environmental and Development Economics. The Ohio State University. E-mail: larson.4@osu.edu e thompson.51@osu.edu.

(Recebido em setembro de 2005. Aceito para publicação em fevereiro de 2007). 


\section{INTRODUÇÃO}

As conseqüências da liberalização comercial e da integração de mercados para países em desenvolvimento têm se tornado importantes temas com as recentes criações de acordos de livre comércio, como o Mercosul nos anos 1990. Espera-se que um acordo de integração regional proporcione aumento e diversificação no comércio entre seus membros. No entanto, a integração que ocorreu com o Mercosul pode ser vista como sendo tímida, principalmente devido à falta de coordenação efetiva e contínua das políticas macroeconômicas adotadas pelos quatro países-membros. Muitos planos econômicos têm sido implementados nestes países desde 1991, objetivando apenas a própria estabilidade econômica de cada país membro, e levantando dúvidas sobre o futuro do Mercosul como um bloco econômico. A ênfase nos programas de estabilização econômica em detrimento de políticas de incentivo ao comércio podem impulsionar a saída de capital para países com menores níveis de tarifas, mas com menores vantagens comparativas. De acordo com Baer et al. (2001), grandes alterações na taxa de câmbio real podem afetar acintosamente retornos de investimentos, resultando em mudanças na localização de novas plantas de produção e/ou realocação da produção das já existentes.

Acredita-se que os diferentes planos econômicos de estabilização adotados ao longo do tempo e implementados pelos países-membros do Mercosul são responsáveis por grande parte da volatilidade da taxa de câmbio real de médio e longo prazos (ER) existente neste bloco. O rationale por trás do que foi exposto acima está no fato de que grandes alteraçóes na taxa de câmbio real, ocasionadas por planos econômicos de estabilização inerentes a cada país, podem aumentar o grau de incerteza entre agentes econômicos domésticos e estrangeiros (parceiros comerciais). De Grauwe e De Bellefroid (1986) atribuem a maior parte desta incerteza às grandes variações na taxa de câmbio real.

A presente pesquisa apresenta algumas características e importantes contribuições que a distinguem de outros estudos, tais como: (i) o nível de desagregação e o tamanho da amostra dos dados a serem utilizados, os quais são maiores que os usados em outros estudos (utiliza-se dados comerciais de dois dígitos SITC ${ }^{\mathrm{l}}$, em contraste ao uso de apenas um dígito SITC como a maior parte dos estudos de comércio); (ii) a análise dos impactos da volatilidade da taxa de câmbio real de médio e longo prazos, em contraste à volatilidade de curto prazo normalmente adotada; (iii) efeitos em nível setorial da volatilidade cambial no comércio brasileiro no Mercosul (a literatura tem mostrado que a ênfase tem sido nos fluxos de comércio agrícola de países desenvol-

1 SITC é a sigla de Classificação Padrão para Comércio Internacional (Standard International Trade Classification). 
vidos); e, finalmente, (iv) o uso de modelo de efeitos fixos (aleatórios) para captar o padrão de comércio brasileiro no Mercosul.

Este estudo investiga como a volatilidade cambial entre países do Mercosul pode levar à devastação do comércio, através da investigação dos principais determinantes do comércio brasileiro no Mercosul. A ênfase está nos diferentes efeitos da volatilidade da taxa de câmbio real de médio e longo prazos em diferentes setores, incluindo o comércio agrícola, pois este é o setor menos protegido nos países da América Latina. De acordo com a literatura empírica, a volatilidade da taxa de câmbio real é responsável por efeitos negativos no comércio agropecuário, conforme Cho et al. (2002) e Maskus (1986).

Um modelo gravitacional é especificado e estimado para avaliar:

(i) os impactos da volatilidade da taxa de câmbio real de médio e longo prazos no comércio em diferentes setores no Mercosul; e

(ii) o padrão de fluxo do comércio brasileiro no Mercosul, procurando quantificar os impactos que mudanças em distância, tarifas e renda têm nos fluxos de comércio.

\section{ESPECIFICAÇÃO DO PROBLEMA}

A criação do Mercosul foi um importante fator na consolidação do processo de abertura da economia brasileira. Os países do Mercosul fecharam um acordo de redução gradual das tarifas para o período entre 1991 e 1994, resultando em um aumento substancial no comércio entre os membros do Mercosul neste período (Tabela 1). Ao mesmo tempo que tanto o comércio quanto a integração dentro do Mercosul aumentaram, os dois maiores países da região, Argentina e Brasil, experimentaram muitas crises domésticas em anos recentes.

TABELA I - TAXA DE CRESCIMENTO MÉDIO ANUAL DO COMÉRCIO BILATERAL ARGENTINA-BR ASIL PARA O PERÍODO 1991-2000

\begin{tabular}{lcccc}
\hline País & $\begin{array}{c}\text { Exportações } \\
\text { Totais }\end{array}$ & $\begin{array}{c}\text { Exportações para o } \\
\text { Mercosul }\end{array}$ & Importações Totais & $\begin{array}{c}\text { Importações do } \\
\text { Mercosul }\end{array}$ \\
\hline Argentina & $8,5 \%$ & $19,0 \%$ & $25,3 \%$ & $30,5 \%$ \\
Brasil & $6,0 \%$ & $22,9 \%$ & $11,8 \%$ & $15,5 \%$ \\
\hline
\end{tabular}

Fonte: Ministério do Desenvolvimento, Indústria e do Comércio; Inter-American Development Bank. 
A falta de coordenação macroeconômica entre os países do Mercosul parece ser uma das muitas causas de divergência e flutuações de precos e taxa cambial, afetando o comércio e a alocação de investimentos. De acordo com Baer et al. (2001), a falta de harmonia nas políticas macroeconômicas afeta o comércio internacional através de dois canais: risco nas transações internacionais e na economia política. O primeiro é caracterizado pelo aumento no risco associado com transações internacionais, afetando as decisões de comércio e resultando em uma alocação de recursos diferente da que seria definida pelas vantagens comparativas. Um aumento na volatilidade da taxa de câmbio real (ER) leva exportadores e importadores aversos ao risco a reduzirem sua oferta e demanda de bens comercializáveis porque eles se deparam com um risco adicional em relação aos lucros externos. O segundo canal, também influenciado pela não-coordenação de políticas, seria o da existência potencial de lobbying para proteger mercados domésticos quando existe um aumento na razão de penetração de bens importados (Trefler, 1993).

Uma consequiência direta do exposto no parágrafo anterior está evidente no Mercosul, cujos membros eliminaram progressivamente a maior parte de suas barreiras comerciais entre 1991 e 1995. No entanto, as tarifas não foram totalmente eliminadas e foi permitido a cada país identificar produtos sensíveis à competição, os quais poderiam ser protegidos até 1999 para a Argentina e Brasil, e até 2001 para Paraguai e Uruguai. ${ }^{2}$ Cada país também podia ter seu próprio nível de tarifa nestes produtos sensíveis. ${ }^{3}$

Assim, é interessante verificar as conseqüências da instabilidade da taxa de câmbio nos diferentes setores do comércio brasileiro no Mercosul. Existem muitos estudos que avaliam a influência da volatilidade da taxa de câmbio na economia de um país. Muitos deles sugerem que a volatilidade da taxa de câmbio reduz o comércio (Hooper e Kohlhagen, 1978; Thursby e Thursby, 1987; Cushman, 1988; Frankel e Wei, 1993; Eichengreen e Irwin, 1995; Rose, 2000). Mas, segundo Sauer e Bohara (2001), a maneira com que fatores, tais como aversão ao risco, oportunidades de hedging, a moeda usada nos contratos e a presença de outros tipos de risco afetam na direção e magnitude entre a incerteza da taxa de câmbio e o comércio é uma questão empírica a ser investigada.

O principal foco deste estudo está em estimar o padrão de fluxo de comércio do Brasil no Mercosul e determinar como o fluxo de comércio responde a mudanças na volati-

2 De acordo com Averbug (1998) e Baer et al. (2001), a lista brasileira era composta por 29 produtos, 212 produtos na lista argentina, 432 na lista paraguaia e 963 na lista uruguaia.

3 As tarifas deveriam convergir para 14\% até janeiro de 2001 para bens de capital, para Argentina e Brasil, e até janeiro de 2006 para Paraguai e Uruguai. Para outros produtos estas tarifas deveriam convergir para 16\% até 2006. No entanto, em meados de julho de 2001, a Argentina reduziu suas tarifas de importação de bens e equipamentos de informática, causando divergências diplomáticas com o Brasil. (Baer et al., 2001) 
lidade da taxa de câmbio e em outros determinantes de comércio, tais como tarifas, distância entre países, PIB e volatilidade da taxa de câmbio de um terceiro país (efeito "third country"). Algumas questôes são abordadas, tais como: o que aconteceria aos fluxos de comércio setoriais se as taxas de câmbio se tornassem mais voláteis? Esta maior volatilidade traria efeitos positivos ou negativos ao comércio brasileiro? Quais seriam as mudanças nos fluxos de comércio como resultado de uma redução nas tarifas ou de um aumento no PIB brasileiro?

\section{REVISÃO DE LITERATURA}

\subsection{Modelos Gravitacionais}

Utiliza-se um modelo gravitacional (Tinbergen, 1962) para determinar empiricamente o padrão de comércio bilateral entre Brasil e seus parceiros de Mercosul. Um modelo gravitacional considera não só fluxos de comércio, mas também efeitos de fronteira (tais como custos de transporte, barreiras comerciais, localização, contigüidade, etc), população, renda nacional e taxa de câmbio. Um modelo gravitacional, ou equação gravitacional, é a solução em forma de equação reduzida de um sistema de equilíbrio geral de comércio internacional em bens finais, o qual assume que o comércio entre dois países é dependente do seu tamanho, estágio de desenvolvimento, grau de abertura de mercado e proximidade. O comércio, assim, é diretamente proporcional ao tamanho do país e inversamente correlacionado com a distância entre os países. Analogamente, o fluxo de comércio entre dois países é uma função da renda, distância e outras variáveis (população, contigüidade, língua, custos de transporte, tarifas etc).

Deardorff (1998) mostra que um modelo gravitacional pode ser consistente com o modelo de Heckscher-Ohlin-Samuelson (HOS) com preferências não-homotéticas, sem a necessidade de se pressupor competição monopolística, como em Bergstrand (1989).

O sucesso dos modelos gravitacionais não pode ser considerado como evidência das teorias do comércio com competição imperfeita e economias de escala como sugerido por Helpman (1987). Deardorff (1998) e Evenett e Keller (2002) ${ }^{4}$ concluem que como a especialização é a "força da gravidade", responsável pelo sucesso empírico dos

4 Este estudo examina as teorias do modelo HOS e a de retornos crescentes de escala para explicar o sucesso empírico da equação gravitacional. Como ambas teorias podem resultar na equação gravitacional, eles estimaram versões puras e híbridas de ambas teorias para dados cross-section para 58 países. Os resultados sugerem que as previsões de um modelo com especialização imperfeita, a qual é baseada nas diferenças na dotação de fatores, suportam empiricamente os dados utilizados. 
modelos gravitacionais, não é necessário identificar um modelo teórico de comércio para derivar a equação gravitacional.

De acordo com Cho et al. (2002), são poucos os estudos que avaliam os impactos da variablidade da taxa de câmbio no comércio agropecuário. Algumas das tentativas iniciais na investigação de tais efeitos são Schuh (1974), Batten e Belongia (1986), Haley e Krissoff (1987), e Bessler e Babula (1987). Alguns estudos analisam os impactos da volatilidade da taxa de câmbio de curto prazo $^{5}$ no comércio agropecuário. Pick (1990) não encontrou evidências de que o risco da taxa de câmbio afeta o comércio dos Estados Unidos com países desenvolvidos, mas encontrou um efeito negativo no fluxo de comércio com países em desenvolvimento. Klein (1990), por sua vez, encontrou impactos negativos da volatilidade da taxa de câmbio de curto prazo no comércio agropecuário dos Estados Unidos. Cho et al. (2002) estimou um modelo gravitacional para muitos países desenvolvidos para investigar os efeitos da volatilidade da ER no comércio agropecuário. Seus resultados sugerem que a incerteza na taxa de câmbio real tem ocasionado impactos negativos no comércio agropecuário para o período de 1974 a 1995.

A competitividade de um país é reduzida com uma supervalorização de sua moeda, e o inverso é verdadeiro quando a moeda é desvalorizada. Conforme Tweeten (1989), a valorização do dólar americano durante os anos 1980 teve impactos negativos nas exportações agrícolas norte-americanas. Cho (2001) argumenta que, devido à redução na competitividade, alguns setores podem perder mercados domésticos e externos, resultando em diminuição nos níveis de emprego e produção. Este resultado contribui para o lobbying por proteção por aqueles grupos que perdem com a valorização da taxa cambial. Se uma medida protecionista é adotada pelo governo após atender aos apelos destes setores, a mesma não é fácil de ser removida quando uma depreciação cambial ocorre. Países que experimentaram grandes variaçóes na taxa de câmbio por longos períodos de tempo são mais propensos a ter reduções no crescimento do comércio (De Grauwe, 1988). Pick e Vollrath (1994) acreditam que movimentos cambiais em países em desenvolvimento têm afetado negativamente a competitividade do setor agropecuário.

\subsection{Efeitos da Volatilidade Cambial em Vários Setores}

A ausência de um sistema cambial estável e bem administrado pode ser uma importante fonte do que é conhecido como "misalignment" (ou "desalinhamento"),

5 Para Peree e Steinherr (1989), a volatilidade da taxa de câmbio de curto prazo é obtida quando se considera a incerteza na taxa de câmbio para um período inferior a um ano.

6 Este termo significa a distância da taxa de câmbio nominal do nível de equilíbrio de longo prazo ou dos fundamentos econômicos. Mais detalhes das conseqüências econômicas do problema de "misalignment" 
principalmente para aqueles países que têm utilizado a paridade de sua moeda com o dólar americano. Argentina e Brasil adotaram regimes cambiais fixos durante os anos 1990, ocasionando substanciais e persistentes desvios das taxas de câmbio nominais dos fundamentos macroeconômicos. O tamanho deste "desalinhamento", no longo prazo, é um importante fator afetando o comércio internacional. O modelo de "bysteresis" oferece uma explicação para esta relação (Baldwin, 1988; Baldwin e Krugman, 1989). O efeito "hysteresis" dos movimentos da taxa de câmbio prevê que um inesperado "desalinhamento" da taxa cambial pode causar uma mudança permanente na estrutura de mercado (Baldwin, 1988; Baldwin e Krugman, 1989). Tanto o modelo "hysteresis" como as evidências empíricas mostram que mudanças na taxa de câmbio trazem impactos diferentes para os diversos setores da economia devido às peculiaridades de cada setor. Estas peculiaridades (ou características) podem ser definidas pelos diferentes níveis de investimento inicial (Baldwin, 1988), pelo nível de substitutabilidade de bens (Dornbusch, 1987), ou se os produtos são ou não duráveis (Froot e Klemperer, 1989).

Devido à grande quantidade de ambigüidades teóricas e empíricas a respeito da relação entre a volatilidade da taxa de câmbio e o comércio, não é surpresa que a literatura empírica sobre este tópico seja tão escassa e limitada. No entanto, o modelo "hysteresis" de Baldwin e Krugman pode ajudar a interpretar os resultados obtidos para os diferentes setores. De acordo com este modelo, setores com demanda por grandes montantes de investimento inicial seriam menos suscetíveis à mudança estrutural induzida pelo choque descrito anteriormente, sofrendo menos com a volatilidade da taxa de câmbio. Por outro lado, aqueles setores que necessitam de baixos valores de investimento tenderiam a ser mais suscetíveis à volatilidade da taxa cambial. Se estes impactos são positivos, negativos, ou neutros, esta é uma questão empírica.

\section{DADOS E PROBLEMAS}

Os dados básicos utilizados consistem de comércio bilateral, e tarifas médias entre Brasil, Argentina, Paraguai e Uruguai, para o período de 1989 a 2002, obtidos a partir do Sistema de Informação e Análise do Comércio (TRAINS)/Solução de Comércio Mundial Integrado (WITS) da UNCTAD. ${ }^{7}$ Este é um banco de dados em painel que consiste de valores nominais de exportaçóes de um país para outro, para diferentes setores (agricultura, indústria química, pecuária, mineração e óleo, manufaturados, e

podem ser encontrados em Tweeten (1989). A volatilidade da taxa de câmbio de longo prazo pode ser considerada como uma proxy do tamanho do "misalignment" (De Grauwe e De Bellefroid, 1986).

7 Conferência das Nações Unidas em Comércio e Desenvolvimento (United Nations Conference on Trade and Development). 
todos os setores simultaneamente), ao nível de dois dígitos SITC. A amostra agregada contém 2.688 observações ( 3 países vezes 64 produtos diferentes vezes 14 anos).

Como este estudo visa investigar os efeitos da variabilidade da taxa de câmbio no comércio brasileiro em nível setorial dentro do Mercosul, os dados foram convertidos na moeda do país exportador através do deflacionamento da taxa de câmbio nominal ${ }^{8}$, pelo índice de preços ao consumidor (CPI) do país exportador. Os valores nominais e reais do PIB (deflacionados pelo CPI), bem como os dados sobre população foram extraídos das Estatísticas Financeiras Internacionais (IFS). Para a variável distância foi definida a distância do "grande círculo" entre os centros econômicos", conforme Soloaga e Winters (2001). As taxas de câmbio real ${ }^{10}$ foram calculadas como:

$$
R E R_{\dot{\mathrm{s}}, t}=N E R_{\dot{\mathrm{b}}, t}\left(\frac{C P I_{s, t}}{C P I_{i, t}}\right)
$$

onde $R E R_{i s, t}$ e $N E R_{i s, t}$ são as taxas de câmbio real e nominal, respectivamente, para o país $i$ com respeito à moeda do país $s$ no período $t$. A expressão (1) mostra como a taxa de câmbio real é calculada para o país $i$ usando o dólar americano de 1995 como a moeda do país $s$ (Estados Unidos). $C P I_{s, t}$ reflete o índice de preços do consumidor nos Estados Unidos no momento $t$. $C P I_{i, t}$ representa o índice de preços ao consumidor no país $i$ no momento $t$. Assim, a taxa de câmbio bilateral real $\left(X_{i j, t}\right)$ para cada país pode ser obtida pelas razões entre cada taxa de câmbio real dos países-membros do Mercosul e a taxa de câmbio real brasileira $(j)$.

A incerteza ${ }^{11}$ de longo prazo da taxa de câmbio é essencial para este estudo. Esta incerteza pode ser obtida através de dois procedimentos usados como proxies da incerteza de longo prazo da taxa de câmbio, o desvio padrão móvel (MSD) e a medida de volatilidade de Peree e Steinherr $(\mathrm{P} \& S) .^{12}$

8 Utilizou-se a informação de taxa de câmbio do final do período, da publicação Estatísticas Financeiras Internacionais (IFS) do Fundo Monetário Internacional (IMF).

9 O método do grande círculo é definido pela média ponderada das latitudes e longitudes dos principais centros econômicos.

10 A principal razão pela qual a taxa de câmbio real é utilizada neste estudo é porque existe a expectativa de que as taxas de câmbio real e nominal estejam altamente correlacionadas e a volatilidade da ER real deve ser maior. De Grauwe e De Bellefroid (1986) sugerem que quando uma moeda sofre desvalorização de determinada proporção, a mudança na taxa de câmbio real é inferior à depreciação inicial, pois a inflação tende a superar a depreciação nominal inicial. Estas diferenças entre ER real e ER nominal podem ser importantes quando as variabilidades de médio e longo prazos são investigadas, que é o caso do presente estudo.

11 Para uma discussão detalhada sobre medidas de volatilidade da taxa de câmbio, ver Lanyi e Suss (1982), Brodsky (1984), e Kenen e Rodrik (1986).

12 Pelo fato de as medidas de volatilidade serem usadas como proxies da incerteza da taxa de câmbio real, volatilidade e incerteza são expressóes usadas para descrever o mesmo fenômeno neste estudo. 
O MSD das diferenças do logaritmo natural da ER bilateral real é uma modificação da medida usualmente utilizada em muitos estudos de dados cross-section ou de séries temporais, tais como Kenen e Rodrik (1986), De Grauwe e De Bellefroid (1986), e Dell'Ariccia (1999). O MSD é usado aqui porque esta medida varia com o tempo, de modo a ser compatível com a característica temporal dos dados em painel disponível, como em Cho et al. (2002).

O MSD das diferenças do logaritmo natural da ER bilateral real $\left(S_{\mathrm{ijt}}\right)$ é dado por:

$$
S_{i j, t}=u_{i j, t}=\sqrt{\frac{\sum_{l=1}^{k}\left(x_{i j, t-l}-\bar{x}_{i j, t}\right)^{2}}{k-1}}
$$

onde $X_{i j, t}$ é a taxa de câmbio bilateral real, $x_{i j, t}=\ln \left(X_{i j, t}\right)-\ln \left(X_{i j, t-1}\right)$, e $k=2,4,6,8$, e $9 \operatorname{anos}^{13} \cdot \bar{x}_{i j, t}$ é a média de $x_{i j, t}$ para os últimos $k$ anos.

A segunda medida de volatilidade da ER real é baseada em Peree e Steinherr (1989), os quais assumem que a incerteza dos agentes econômicos é definida pelas experiências passadas a respeito dos valores máximo e mínimo da taxa de câmbio, as quais são ajustadas pela experiência do ano anterior relativa a uma taxa de câmbio de "equilíbrio". Deste modo, grandes alterações cambiais ocorridas no passado acabam gerando a volatilidade esperada. Estes autores propóem a seguinte medida da incerteza da taxa de câmbio:

$$
V_{i j . t}=u_{i j, t}=\frac{\max X_{i j, t-k}^{t}-\min X_{i j, t-k}^{t}}{\min X_{i j, t-k}^{t}}+\left[1+\frac{\left|X_{i j, t}-X_{i j, t}^{k}\right|}{X_{i j, t}^{k}}\right]
$$

onde $k$ é a duração do período; min $X_{i j, t}^{t}$ é o valor absoluto mínimo da taxa de câmbio real nos últimos $k$ períodos; max $X_{i j, t}^{t}$ é o valor absoluto máximo da taxa de câmbio real nos últimos $k$ períodos; $X_{i j, t}^{k}$ é a média dos valores absolutos da taxa de câmbio real para os últimos $k$ períodos. Este valor é uma proxy da taxa de câmbio real de equilíbrio de longo prazo. ${ }^{14}$ Cada período $k$ nesta análise é representado por cada ano. A justificativa está na ênfase dada aos efeitos de médio a longo prazos da incerteza da taxa de câmbio real.

As Figuras 1 e 2 mostram as duas medidas da volatilidade da taxa de câmbio real. Segundo a medida MSD, a volatilidade da ER real entre o peso argentino e o real

13 O período de tempo é arbitrariamente escolhido para investigar a robustez dos resultados.

14 De acordo com Mark (1995), não é possível se obter uma medida da taxa de câmbio de longo prazo. Por esta razão, este estudo adota a média aritmética da taxa de câmbio real para todo o período disponível da amostra de modo a se obter uma aproximação de tal medida de equilíbrio. 
brasileiro tem, relativamente, alto grau de volatilidade. No entanto, esta volatilidade sofreu forte redução após 1997 devido à depreciação da moeda brasileira. Nota-se que a volatilidade do guarani paraguaio/real brasileiro é a mais estável no Mercosul. A relativa estabilidade da volatilidade da ER para o período 1992 a 1997 é devida, principalmente, à política cambial adotada pelo Brasil, utilizando esta política como âncora para controle da inflação interna (Figura 1).

\section{FIGURA 1 - VOLATILIDADE DA TAXA DE CAMMBIO REAL NO MERCOSUL,} MEDIDA PELO DESVIO PADR ÃO MÓVEL (MSD), 1989 - 2002

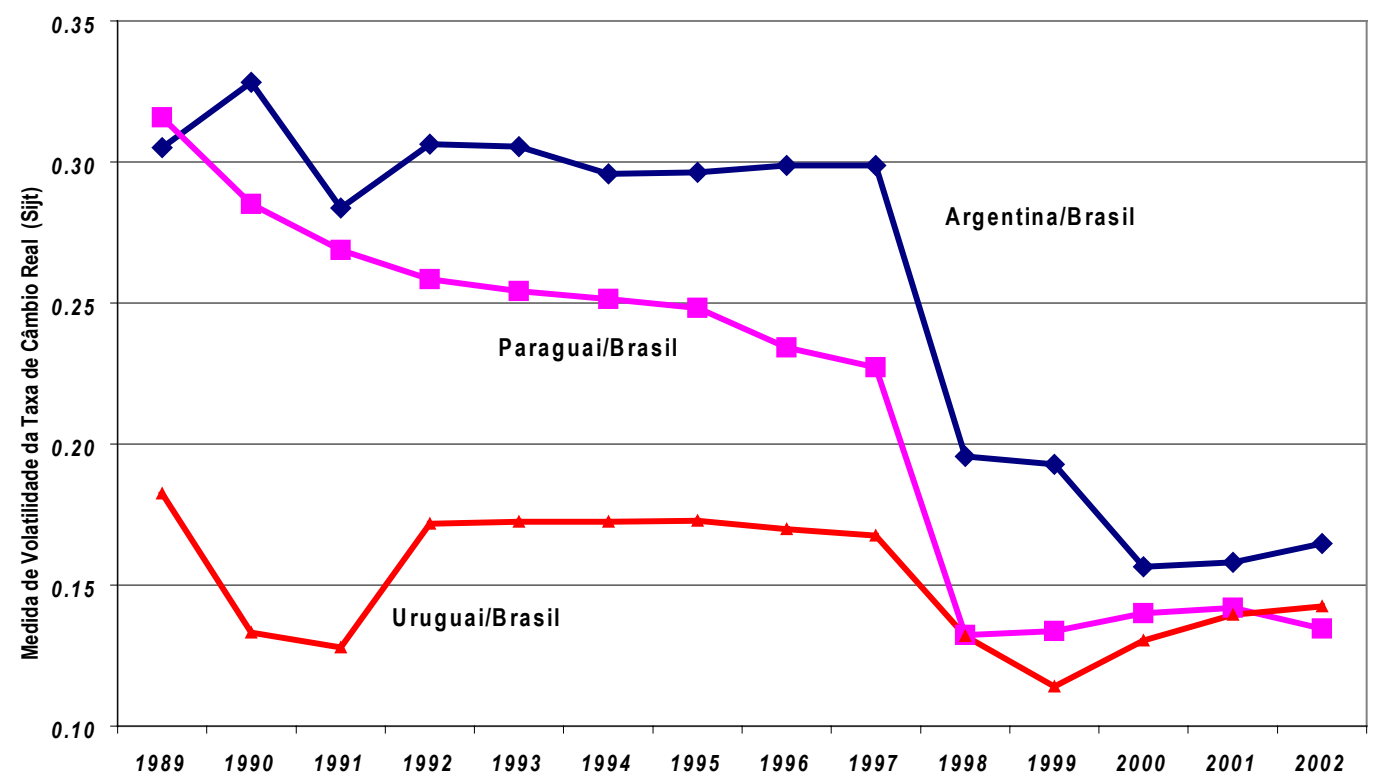

A medida P\&S da volatilidade da ER (Figura 2) é caracterizada por um comportamento decrescente no período 1990 a 1999, provavelmente devido à "experiência acumulada", característica desta medida de volatilidade, a qual leva em conta valores da taxa de câmbio vigentes no passado. ${ }^{15}$ Após a grande desvalorização cambial brasileira em 1999, a volatilidade da ER cresceu bastante, principalmente para a volatilidade peso argentino/real brasileiro, relação esta que se tornou a mais volátil entre os países do Mercosul após a implementação do Plano Real, em meados de 1994. Em geral, a medida P\&S se mostrou mais volátil que a medida MSD.

15 As taxas de inflação estiveram bastante altas nestes países no início dos anos 90, também afetando o comportamento das taxas de câmbio real neste período. A relação entre taxas de inflação e taxas de câmbio pode ser ilustrada através do modelo monetário da taxa de câmbio (Mark, 1995). 
FIGURA 2 - VOLATILIDADE DA TAXA DE CÂMBIO REAL (MEDIDA DE PEREE E STEINHERR) NO MERCOSUL, 1989 - 2002

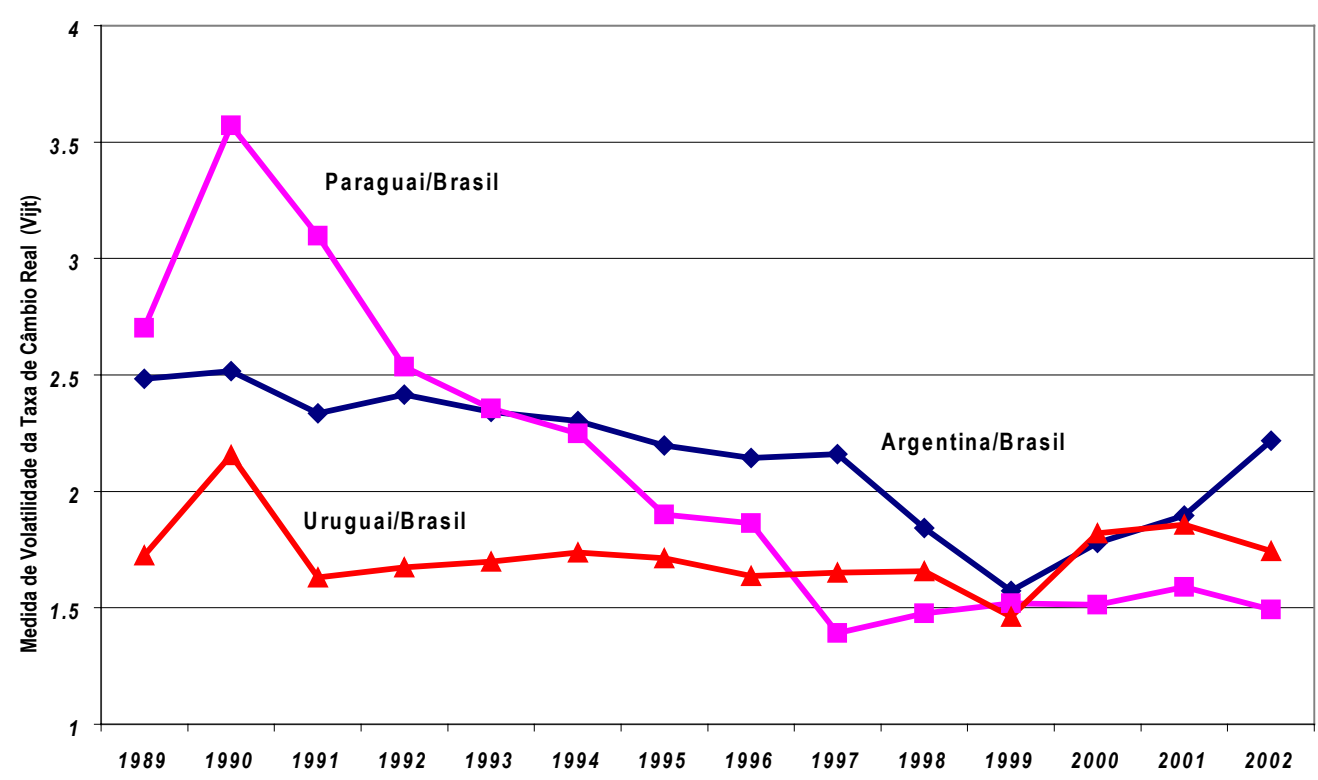

Existem dois outros problemas importantes. O primeiro é a potencial presença de viés de simultaneidade, devido à endogeneidade da taxa de câmbio decorrente das ações de um banco central na tentativa de estabilizar os termos de troca entre os parceiros comerciais do Mercosul. A volatilidade da taxa de câmbio e o fluxo de comércio continuariam negativamente correlacionados, mas a direção de causalidade poderia ser ambígua. Para lidar com este problema, utiliza-se o procedimento de variável instrumental para testar a presença de viés de simultaneidade através do teste de Hausman (Dell'Ariccia, 1999). Mas a pergunta relevante é: qual variável instrumental pode ser usada para a volatilidade da ER real? Neste estudo adota-se o instrumento para a volatilidade da ER sugerido por Bittencourt (2004), cujo resultado é representado pela equação (4).

$s_{t}=\frac{1}{1+\lambda} f_{t}+\frac{\lambda}{1+\lambda} E_{t} s_{t+1}=\frac{1}{1+\lambda} \sum_{j=0}^{k}\left(\frac{\lambda}{1+\lambda}\right)^{j} E_{t} f_{t+j}+\left(\frac{\lambda}{1+\lambda}\right)^{k+1} E_{t} s_{t+k+1}$

onde $f$ representa os fundamentos econômicos, $\lambda$ é a semi-elasticidade da demanda por moeda em relação à taxa de juros, e $s$ é a taxa de câmbio. 
A variabilidade dos fundamentos é obtida através do desvio padrão móvel dos fundamentos, usando como defasagens quatro e oito anos. As variáveis usadas para construir os fundamentos são a oferta real de moeda ${ }^{16}$ e o PIB real para cada país.

O segundo problema é o efeito da volatilidade da ER real de um terceiro país no comércio bilateral em análise. O efeito "third country" foi investigado por Wei (1996), Dell'Ariccia (1999), e Cho et al. (2002), usando uma medida que leva em conta a volatilidade da ER real para todos os outros países, exceto os dois países envolvidos no comércio em análise. No entanto, o procedimento a ser adotado neste estudo é um pouco diferente dos estudos anteriores, os quais utilizaram as participações dos países no comércio total como ponderações para obter a medida de volatilidade do efeito "third country". O presente estudo propõe uma diferenciação nas ponderações por setores da economia, ou seja, leva em conta as participações no comércio específicas a cada setor como ponderações. Além disso, estudos anteriores consideraram as participações dos países no comércio total baseadas em um único ano, sob a justificativa de que tais participações são relativamente constantes ao longo do tempo. $\mathrm{O}$ procedimento sugerido aqui é o de se utilizar como ponderações as participações no comércio por setores para cada ano disponível pelos dados, pois algumas mudanças podem ter ocorrido de um ano para outro na amostra, caracterizando diferentes respostas dos fluxos de comércio em nível setorial a movimentos cambiais.

A medida de volatilidade da ER real de um terceiro país, volatilidade "third country", $\left(u 3_{i j, t}\right)$ é dada por:

$$
u 3_{i j, t}^{g}=\sum_{i \neq j} u_{i j, t} w_{i j, t}^{g}+\sum_{j \neq i} u_{j i, t} w_{j i, t}^{g}
$$

Onde $u_{i j, t}\left(u_{j i, t}\right)$ é a medida de volatilidade da ER real, seja ela a medida de desvio padrão móvel $\left(S_{\mathrm{ij}, \mathrm{t}}\right)$ ou a medida de Peree e Steinherr $\left(\mathrm{V}_{\mathrm{ij}, \mathrm{t}}\right)$, definidas pelas equações (2) e (3); $g=1, \ldots, 5$, onde 1 é para o setor pecuário; 2 para o setor agrícola; 3 para o setor de produtos químicos; 4 para o setor de manufaturados; e 5 para o setor de mineração e óleo; $w_{i j, t}{ }^{g}$ e $w_{j i, t}{ }^{g}$ são as participações no comércio específicas a cada setor dos outros países. Espera-se que o sinal do coeficiente para o efeito "third country" no comércio seja positivo, como o obtido por Wei (1996). No entanto, para Dell'Ariccia (1999) esta correlação foi negativa e não significativa, e para Cho et al. (2002) este coeficiente foi positivo e negativo para diferentes setores.

16 A oferta real de moeda foi obtida através da soma da moeda fora dos bancos "currency money" mais a demanda por depósitos deflacionados para a moeda do país, e então convertidos para o dólar americano de 1995. 


\section{ESPECIFICAÇÃO DO MODELO ECONOMÉTRICO}

A equação gravitacional a ser estimada é especificada econometricamente como:

$$
\begin{aligned}
& \ln T_{i j, t}^{g}=\alpha_{i}^{g}+\gamma_{1}^{g} \ln \left(Y_{i t} Y_{j t}\right)+\gamma_{2}^{g}\left(\text { Pop }_{i t} \text { Pop }_{j t}\right)+\gamma_{3}^{g}\left(u_{i j, t}\right)+\gamma_{4}^{g} \ln \left(D_{i j}\right)+ \\
& \gamma_{5}^{g} \ln \left(1+\text { Tariff }_{i j, t}^{g}\right)+\gamma_{6}^{g}\left(u 3_{i j, t}\right)+\varepsilon_{i j, t}^{g}
\end{aligned}
$$

onde $T_{i j, t}{ }^{g}$ é o comércio bilateral entre países $i$ e $j$ em cada setor $g ; Y_{i t} Y_{j t}$ é o produto dos PIB's dos dois países no período $t$, e seu coeficiente é esperado ser positivo. Pop ${ }_{i t} P_{o p}$ é o produto entre as populações dos dois países no período $t$, o qual pode-se assumir que reduz o comércio à medida que a população nos dois países cresce. Desde que a demanda por produção doméstica apresente crescimento, haverá uma redução natural da quantidade disponível para comércio. Espera-se que o sinal do coeficiente desta variável seja negativo. A variável $u_{i j, t}$ é a medida de volatilidade da ER real, seja medida por MSD $\left(S_{i j, t}\right)$ ou por P\&S $\left(V_{i j, t}\right)$, definida anteriormente pelas equações $(2)$ e (3); espera-se que seu coeficiente seja negativo. $D_{i j}$ é a distância entre países $i$ e $j$, a qual representa uma proxy para os custos de transporte e deve afetar negativamete o comércio bilateral. ${ }^{17}$ Tariff é a simples média das tarifas entre os países $i$ e $j$ dentro de cada categoria de produtos; espera-se que seu coeficiente seja negativo, resultando em maior comércio à medida que as tarifas são reduzidas. A variável $u 3_{i j, t}$ é a volatilidade da ER real de um terceiro país (efeito "third country") para todos os países exceto os países $i$ e $j$ analisados; o sinal do coeficiente desta variável é ambíguo, conforme Wei (1996) e Cho et al. (2002).

A equação gravitacional (6) será estimada sob duas especificações distintas da medida de volatilidade da ER real $\left(\mathrm{u}_{\mathrm{ij}, \mathrm{t}}\right)$ a ser usada: a medida MSD $\left(S_{i j, t}\right)$ e a medida P\&S $\left(V_{i j, t}\right)$.

De acordo com Egger (2002), a escolha do modelo econométrico é de grande relevância para a determinação dos fluxos de comércio bilateral. Neste estudo, o procedimento de estimação a ser adotado será o da econometria de dados em painel. As vantagens deste procedimento estão na obtenção de estimativas mais confiáveis, na redução de problemas de multicolinearidade, maior número de graus de liberdade,

17 Linnemann (1966) aponta que o efeito da distância no comércio vem de três fontes:1) custos de transporte; 2 ) tempo (perecibilidade, adaptação às condições de mercado, irregularidades na oferta, custos de juros); e 3) distância "psíquica", a qual inclui familiaridades com as leis, instituições e cultura. A idéia de Linnemann sobre o significado mais abrangente da variável distância é apontada também por Frankel et al. (1998), o qual notou que os custos de envio físico de mercadorias podem não ser os custos mais importantes que compóem os custos associados com a distância. Custos de transporte devem ser vistos como custos de transação, os quais incluem não só o custo de transporte físico de bens, mas também os custos de comunicação e o fato de que os países tendem a ter um melhor conhecimento das instituições e vizinhos mais próximos. 
além de permitir a inclusão da volatilidade da ER real no modelo, o que não seria possível em uma análise cross-section. ${ }^{18}$

\section{RESULTADOS E DISCUSSÃO}

Este estudo procura captar os efeitos de médio e longo prazos da volatilidade da ER real no comércio setorial brasileiro e determinar os impactos de outros fatores importantes que contribuem para este comércio. Os resultados sob diferentes períodos de defasagens e com uso de variáveis instrumentais não são reportados devido às restrições de espaço.

Os resultados econométricos a seguir referem-se a cada um dos cinco setores, além da consideração de todos os setores conjuntamente. Os resultados mostram que o comércio brasileiro é negativamente afetado não somente por movimentos na taxa cambial brasileira, mas também nas taxas de seus parceiros de Mercosul. A variável população não foi incluída nas estimações finais devido à sua alta correlação com a renda (PIB).

Os resultados se mostraram diferentes de acordo com a especificação de volatilidade da ER usada (Tabela 2). De acordo com o teste de Hausman, os estimadores de variáveis instrumentais não se mostraram superiores aos obtidos através do modelo de efeitos fixos (aleatórios). ${ }^{19}$

A variável PIB tem um importante papel no comércio agrícola, com um coeficiente de 4,63 na especificação MSD (Tabela 2). Um aumento de $1 \%$ na renda de cada país (Brasil e seus parceiros) aumenta o comércio em 4,63\%. Tanto tarifas como volatilidade da ER real apresentaram coeficientes significativos, mas de maior magnitude na especificação MSD. Estes coeficientes afetam negativamente o comércio bilateral agrícola, sugerindo que a falta de políticas macroeconômicas estáveis pode reduzir o comércio bilateral no Mercosul. Com relação ao efeito "third country", o coeficiente associado foi significativo estatisticamente somente na especificação MSD, com sinal positivo e relativamente grande. Apesar de o sinal esperado para este coeficiente poder ser ambíguo, (Wei, 1996; Cho et al., 2002), o mesmo parece indicar que maior volatilidade da ER real de um terceiro país do Mercosul contribui para aumentar o

18 Cho (2001) inclui como variáveis a taxa de câmbio real e a volatilidade em suas abordagens de cross-section e painel, respectivamente. No entanto, a inclusão da taxa de câmbio real na estimação cross-section não faz sentido, pois esta não traz nenhuma informação se a moeda sob análise está desvalorizada ou valorizada.

19 Em cada estimação, referente a cada setor, utilizou-se o teste de Hausman para decidir se o modelo de efeitos fixos se mostra superior ao de efeitos aleatórios, e os resultados reportados nas tabelas a seguir já são decorrentes deste teste. 
comércio entre Brasil e um parceiro qualquer deste bloco no setor agrícola. Como o esperado, os resultados do modelo de efeitos aleatórios para a especificação $P \& S$ mostram um coeficiente estimado negativo para a variável distância e significativo estatisticamente ao nível de $10 \% .{ }^{20}$

TABELA 2 - ESTIMAÇÓES DOS EFEITOS FIXOS E ALEATÓRIOS PARA O COMÉRCIO AGRÍCOLA ENTRE BRASIL E SEUS PARCEIROS DO MERCOSUL, 1989 - 2002, POR MEDIDA DE VOLATILIDADE DA TAXA DE CÂMBIO

\begin{tabular}{lcc}
\hline \multirow{2}{*}{ Variável } & \multicolumn{2}{c}{ Medida de volatilidade da taxa de câmbio } \\
\cline { 2 - 3 } & \multicolumn{1}{c}{ Especificação MSD (FE) } & Especificação P\&S (RE) \\
\hline \multirow{2}{*}{ PIB } & $4,63^{*}$ & $1,84^{* *}$ \\
& $(6,29)$ & $(2,51)$ \\
Distância & - & $-4,83^{* * *}$ \\
Tarifa média & $-7,43^{*}$ & $(-1,82)$ \\
& $(-6,49)$ & $-4,97^{*}$ \\
Volatilidade da ER real & $-3,22^{* *}$ & $(-4,12)$ \\
\multirow{2}{*}{ Efeito "third country" } & $(-2,41)$ & $-0,59^{* *}$ \\
& $12,74^{*}$ & $(-2,24)$ \\
& $(5,76)$ & $-0,13$ \\
& $\mathrm{t}=14 ; \mathrm{n}=676 ; \mathrm{i}$ (grupos de produtos) $=17$ & $(-0,33)$ \\
\hline
\end{tabular}

Nota: Todos os valores entre parênteses são valores $\mathrm{t} e \mathrm{z}$.

$\left(^{*}\right)$ Estatisticamente significativo ao nível de 1\%; $\left(^{* *}\right)$ Estatisticamente significativo ao nível de 5\%; $\left(^{* *}\right)$ Estatisticamente significativo ao nível de $10 \%$.

FE é o modelo de efeitos fixos e RE é o modelo de efeitos aleatórios.

Os resultados para o setor pecuário (Tabela 3) mostram que os principais fatores determinantes do comércio neste setor são as tarifas e o PIB, pois seus coeficientes foram os únicos significativos estatisticamente. As medidas de volatilidade da ER real (MSD e P\&S) produziram coeficientes não-significativos. A variável volatilidade da ER real pareceu não ser importante para o comércio neste setor, e este resultado mostrou-se robusto para diversas especificações com diferentes períodos de tempo.

20 O uso de diferentes períodos para as variáveis volatilidade da ER na especificação MSD foi consistente com os resultados apresentados na Tabela 2. As principais alterações ocorreram com relação à magnitude do coeficiente do PIB e com a significância estatística do coeficiente da volatilidade da ER real. Para períodos específicos, o coeficiente estimado do PIB foi pequeno, mas significativo, e o coeficiente da volatilidade da ER real foi não significativo. A especificação P\&S mostrou-se mais robusta sob diferentes especificações de períodos considerados para captar a volatilidade da ER, e alguns períodos mostraram um efeito "third country" negativo e significativo, em contraste com os resultados obtidos através da especificação MSD. 
TABELA 3 - ESTIMAÇÕES DOS EFEITOS FIXOS PARA O COMÉRCIO PECUÁRIO ENTRE BRASIL E SEUS PARCEIROS DO MERCOSUL, 1989 - 2002, POR MEDIDA DE VOLATILIDADE DA TAXA DE CÂM$\mathrm{BIO}$

\begin{tabular}{lcc}
\hline \multirow{2}{*}{ Variável } & \multicolumn{2}{c}{ Medida de volatilidade da taxa de câmbio } \\
\cline { 2 - 3 } & \multicolumn{1}{c}{ Especificação MSD (FE) } & Especificação P\&S (FE) \\
\hline \multirow{2}{*}{ PIB } & $1,02^{*}$ & $1,09^{*}$ \\
\multirow{2}{*}{ Tarifa média } & $(2,73)$ & $(2,95)$ \\
& $-5,77^{* *}$ & $-6,22^{*}$ \\
Volatilidade da ER real & $(-1,97)$ & $(-2,11)$ \\
& 0,30 & 0,10 \\
Efeito "third country" & $(0,08)$ & $(0,24)$ \\
& 0,49 & 0,29 \\
& $(0,14)$ & $(0,46)$ \\
\hline
\end{tabular}

Nota: Todos os valores entre parênteses são valores $\mathrm{t}$.

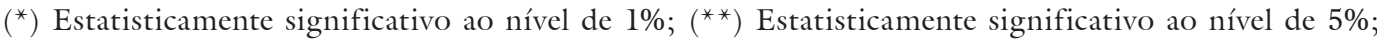
$\left({ }^{* *}\right)$ Estatisticamente significativo ao nível de $10 \%$.

FE é o modelo de efeitos fixos.

O comércio brasileiro no setor pecuário parece ser muito sensível a mudanças nas tarifas e nos níveis de renda do Brasil e de seus parceiros comerciais. ${ }^{21}$ De acordo com os resultados obtidos, uma redução de $1 \%$ nas tarifas aumentaria o comércio neste setor em $6 \%$, aproximadamente. No entanto, uma redução na volatilidade da ER real, através de maior coordenação das políticas macroeconômicas, não parece contribuir para um aumento no comércio entre os países do Mercosul.

Os resultados para os setores químico e agrícola foram diferentes em termos de magnitude e significância estatística das suas estimações (Tabelas 2 e 4). Os coeficientes do PIB e da volatilidade da ER real foram siginificativos estatisticamente nos dois setores. É interessante notar que, em valores absolutos, todos os coeficientes estimados sob a especificação MSD foram maiores que os obtidos com a especificação P\&S. No caso do setor de produtos químicos, os resultados da especificação $P \& S$ mostraram que, apesar da variável distância não ser importante no comércio deste setor, o efeito

21 Os resultados para o setor pecuário também foram robustos quando comparados com as estimações usando variáveis instrumentais (não reportadas), tais como os fundamentos econômicos, e seus desvios padrão móveis com períodos de quatro e oito anos. 
“third country" é importante obstáculo ao comércio no Mercosul. ${ }^{22}$ De acordo com a especificação MSD, uma redução de $10 \%$ na volatilidade da ER real implicaria um aumento no comércio de $10,9 \%{ }^{23}$ No caso da especificação P\&S, esta mesma redução na volatilidade cambial resultaria em um crescimento de $21 \%$ no comércio (Tabela 4).

TABELA 4 - ESTIMAÇÓES DOS EFEITOS FIXOS E ALEATÓRIOS PARA O COMÉRCIO DE PRODUTOS QUÍMICOS ENTRE BRASIL E SEUS PARCEIROS DO MERCOSUL, 1989 - 2002, POR MEDIDA DE VOLATILIDADE DA TAXA DE CÂMBIO

\begin{tabular}{lcc}
\hline \multirow{2}{*}{ Variável } & \multicolumn{2}{c}{ Medida de volatilidade da taxa de câmbio } \\
\cline { 2 - 3 } & \multicolumn{1}{c}{ Especificação MSD (FE) } & Especificação P\&S (RE) \\
\hline \multirow{2}{*}{ PIB } & $1,05^{*}$ & $0,80^{*}$ \\
\multirow{2}{*}{ Distância } & $(4,76)$ & $(3,92)$ \\
\multirow{2}{*}{ Tarifa média } & - & $-0,67$ \\
\multirow{2}{*}{ Volatilidade da ER real } & $-2,61^{* * *}$ & $(-0,92)$ \\
\multirow{2}{*}{ Efeito "third country" } & $(-1,90)$ & 0,93 \\
& $-5,81^{*}$ & $(0,76)$ \\
& $(-4,24)$ & $-1,09^{*}$ \\
& $-2,47$ & $(-4,54)$ \\
& $(-1,13)$ & $-1,32^{*}$ \\
\hline
\end{tabular}

Nota: Todos os valores entre parênteses são valores t e $\mathrm{z}$.

$\left(^{*}\right)$ Estatisticamente significativo ao nível de 1\%; $\left(^{* *}\right)$ Estatisticamente significativo ao nível de 5\%; $\left(^{* *}\right)$ Estatisticamente significativo ao nível de $10 \%$.

FE é o modelo de efeitos fixos e RE é o modelo de efeitos aleatórios.

O maior setor individual analisado foi o setor manufatureiro (23 diferentes categorias de produtos), com 905 observações. O modelo de efeitos fixos foi estimado para am-

22 Os resultados para ambas especificações foram consistentes sob diferentes períodos de tempo. No entanto, quando se utilizou um período de tempo de seis anos, os coeficientes estimados para tarifas e efeito "third country" tornaram-se significativos. O teste de Hausman não rejeitou os coeficientes estimados pelo modelo de efeitos fixos (aleatórios) como sendo não-tendenciosos e consistentes, em comparação com os obtidos através do uso de variáveis instrumentais.

23 A média da volatilidade da ER real usada para obter esta interpretação foi 0,189 e 1,99, respectivamente, para as especificações MSD e P\&S. É importante lembrar que para obter a interpretação de elasticidade do efeito da volatilidade cambial no comércio através do modelo utilizado, deve-se multiplicar o coeficiente estimado pelo valor médio da medida de volatilidade utilizada na estimação. 
bas especificações de medidas de volatilidade, MSD e P\&S, pois o modelo de efeitos aleatórios foi rejeitado através do teste de Hausman. ${ }^{24}$

Apesar de o PIB afetar positivamente o comércio de manufaturados, este comércio é inversamente relacionado com tarifas, volatilidade da ER real e efeito "third country" (Tabela 5). Os coeficientes estimados pela especificação MSD foram maiores que os obtidos pela especificação P\&S, e a única exceção foi o coeficiente do efeito "third country". As estimativas mostram que com uma redução de $1 \%$ na volatilidade da ER real o comércio cresceria cerca de $1,6 \%$ e $1,1 \%$, respectivamente, de acordo com as especificações MSD e P\&S. ${ }^{25}$ Os coeficientes estimados para as variáveis volatilidade da ER real e efeito "third country" parecem enfatizar a idéia de que a busca por maior coordenação entre as políticas macroeconômicas entre os países do Mercosul pode reduzir os efeitos adversos da volatilidade cambial no comércio manufatureiro entre estes parceiros comerciais.

TABELA 5 - ESTIMAÇÓES DOS EFEITOS FIXOS PARA O COMÉRCIO DE MANUFATURADOS ENTRE BRASIL E SEUS PARCEIROS DO MERCOSUL, 1989 - 2002, POR MEDIDA DE VOLATILIDADE DA TAXA DE CÂMBIO

\begin{tabular}{lcc}
\hline & \multicolumn{2}{c}{ Medida de volatilidade da taxa de câmbio } \\
\cline { 2 - 3 } Variável & Especificação MSD (FE) & Especificação P\&S (FE) \\
\hline \multirow{2}{*}{ PIB } & $1,94^{*}$ & $1,48^{*}$ \\
\multirow{2}{*}{ Tarifa média } & $(13,64)$ & $(11,37)$ \\
& $-4,94^{*}$ & $-4,04^{*}$ \\
Volatilidade da ER real & $(-5,71)$ & $(-4,56)$ \\
\multirow{2}{*}{ Efeito "third country" } & $-7,74^{*}$ & $-0,56^{*}$ \\
& $(-5,36)$ & $(-3,34)$ \\
& $-0,40$ & $-1,44^{*}$ \\
& $(-0,28)$ & $(-5,94)$ \\
\hline
\end{tabular}

Nota: Todos os valores entre parênteses são valores $\mathrm{t}$.

$\left(^{*}\right)$ Estatisticamente significativo ao nível de $1 \% ;\left(^{* *}\right)$ Estatisticamente significativo ao nível de 5\%; $\left({ }^{* *}\right)$ Estatisticamente significativo ao nível de $10 \%$.

FE é o modelo de efeitos fixos.

24 Os resultados (Tabela 5) foram robustos para diferentes combinaçóes de períodos de tempo para ambas especificações de medidas de volatilidade usadas nas estimações. O uso de variáveis instrumentais para as medidas de volatilidade não produziram melhores resultados dos que os obtidos aqui.

25 Para interpretar o impacto da volatilidade da ER real no comércio total como se interpreta a elasticidade, é necessário usar a média das medidas MSD e P\&S, cujos valores são, respectivamente, 0,27 e 1,97. 
Os resultados para o setor de mineração e óleo mostram que o comércio neste setor não responde a alterações nos níveis de tarifas (Tabela 6). O coeficiente relativo à variável tarifa média não foi significativo para nenhuma especificação de medida de volatilidade da ER real. O PIB foi, mais uma vez, importante determinante do comércio bilateral também neste setor. De acordo com ambas especificações, um aumento de 10 $\%$ no nível de renda do Brasil e seus parceiros traria aumentos entre 11 e 16,5 \% no volume comercializado neste setor. Os coeficientes da volatilidade da ER real e efeito "third country" se mostraram negativamente correlacionados com o comércio bilateral. Estes coeficientes estimados apresentaram maior magnitude na especificação MSD do que na P\&S, quando comparados com os resultados obtidos de outros setores. ${ }^{26}$

TABELA 6 - ESTIMAÇÕES DOS EFEITOS FIXOS E ALEATÓRIOS PARA O COMÉRCIO DO SETOR DE MINERAÇÃO E ÓLEO ENTRE BRASIL E SEUS PARCEIROS DO MERCOSUL, 1989 - 2002, POR MEDIDA DE VOLATILIDADE DA TAXA DE CÂMBIO

\begin{tabular}{lcc}
\hline \multirow{2}{*}{ Variável } & \multicolumn{2}{c}{ Medida de volatilidade da taxa de câmbio } \\
\cline { 2 - 3 } & \multicolumn{1}{c}{ Especificação MSD (FE) } & Especificação P\&S (RE) \\
\hline \multirow{2}{*}{ PIB } & $1,65^{*}$ & $1,11^{*}$ \\
\multirow{2}{*}{ Distância } & $(7,13)$ & $(4,74)$ \\
\multirow{2}{*}{ Tarifa média } & - & $-1,86^{* *}$ \\
\multirow{2}{*}{ Volatilidade da ER real } & 1,88 & $(-0,91)$ \\
\multirow{2}{*}{ Efeito "third country" } & $(0,78)$ & 3,02 \\
& $-7,79^{*}$ & $(1,24)$ \\
& $(-3,00)$ & $-0,89^{*}$ \\
& $-2,76$ & $(-2,65)$ \\
& $(-1,13)$ & $-1,36^{*}$ \\
\hline
\end{tabular}

Nota: Todos os valores entre parênteses são valores t e z.

${ }^{*}$ Estatisticamente significativo ao nível de 1\%; ${ }^{* *}$ Estatisticamente significativo ao nível de 5\%; $\left(*^{* *}\right)$ Estatisticamente significativo ao nível de $10 \%$.

FE é o modelo de efeitos fixos e RE é o modelo de efeitos aleatórios.

O último conjuto de estimações considerou todos os setores conjuntamente, o qual foi chamado "comércio total" (Tabela 7). Os resultados mostram que todos os coefi-

26 Apesar de não reportados, os resultados para o setor de mineração e óleo foram robustos para diferentes períodos de tempo e, mais uma vez, o modelo com inclusão de variáveis instrumentais foi rejeitado pelo teste de Hausman. 
cientes estimados foram significativos estatisticamente ao nível de significância de 1\%, e os mesmos apresentaram os sinais esperados em ambas especificações de medidas de volatilidade adotadas. ${ }^{27}$ Não foi surpresa que, mais uma vez, a principal diferença entre as especificações tenha sido a maior magnitude dos coeficientes obtidos pela especificação MSD. A volatilidade cambial presente nos países do Mercosul parece ser um obstáculo para o comércio total entre estes países. Uma redução de $10 \%$ no efeito "third country" indica um aumento no comércio total de $4,4 \%$ e $20 \%$, respectivamente, segundo as especificações MSD e P\&S. ${ }^{28}$

TABELA 7 - ESTIMAÇÓES DOS EFEITOS FIXOS PARA O COMÉRCIO TOTAL ENTRE BRASIL E SEUS PARCEIROS DO MERCOSUL, 1989 - 2002, POR MEDIDA DE VOLATILIDADE DA TAXA DE CAMMBIO

\begin{tabular}{lcc}
\hline \multirow{2}{*}{ Variável } & \multicolumn{2}{c}{ Medida de volatilidade da taxa de câmbio } \\
\cline { 2 - 3 } & \multicolumn{1}{c}{ Especificação MSD (FE) } & Especificação P\&S (FE) \\
\hline \multirow{2}{*}{ PIB } & $1,24^{*}$ & $1,15^{*}$ \\
\multirow{2}{*}{ Tarifa média } & $(11,29)$ & $(13,25)$ \\
& $-4,55^{*}$ & $-3,02^{*}$ \\
Volatilidade da ER real & $(-7,43)$ & $(-5,01)$ \\
\multirow{2}{*}{ Efeito "third country" } & $-5,88^{*}$ & $-0,65^{*}$ \\
& $(-7,08)$ & $(-5,94)$ \\
& $-2,40^{*}$ & $-0,99^{*}$ \\
& $(-5,69)$ & $(-6,62)$ \\
\hline
\end{tabular}

Nota: Todos os valores entre parênteses são valores t.

$\left(^{*}\right)$ Estatisticamente significativo ao nível de 1\%; $\left(^{* *}\right)$ Estatisticamente significativo ao nível de 5\%; $\left(^{* *}\right)$ Estatisticamente significativo ao nível de $10 \%$.

FE é o modelo de efeitos fixos.

A Tabela 8 reporta os resultados obtidos usando as medidas MSD e P\&S de volatilidade da ER real. Os resultados para a volatilidade da ER real foram bastante diferentes em termos de magnitude para todos os setores analisados sob a especificação MSD, exceto para os setores de manufaturados, e de mineração e óleo, os quais apresentaram coeficientes elevados para esta variável. No setor pecuário, a volatilidade da ER real não se mostrou uma importante variável para explicar o padrão de comércio deste setor entre os países do Mercosul. A volatilidade da ER real não só parece ser muito

27 Os resultados foram robustos para medidas de volatilidade especificadas sob diferentes períodos de tempo (não reportadas). Não só o teste de Hausman não foi significativo em rejeitar o modelo de efeitos fixos, como também o modelo com variáveis instrumentais não se mostrou superior ao modelo de efeitos fixos.

28 Os valores médios para o efeito "third country" usados para obter esta interpretação foram 0,185 e 2,02, respectivamente, para as especificações MSD e P\&S. 
importante para o setor de produtos químicos, como também apresentou a maior magnitude sob a especificação $P \& S$ dentre os setores analisados.

TABELA 8 - PRINCIPAIS COEFICIENTES SIGNIFICATIVOS PARA O COMÉRCIO SETORIAL ENTRE BRASIL E SEUS PARCEIROS DO MERCOSUL, 1989 - 2002, POR MEDIDA DE VOLATILIDADE DA TAXA DE CÂMBIO

\begin{tabular}{|c|c|c|c|c|}
\hline \multirow[b]{3}{*}{ Setores } & \multicolumn{4}{|c|}{ Medida de volatilidade da taxa de câmbio } \\
\hline & \multicolumn{2}{|c|}{ Especificação MSD } & \multicolumn{2}{|c|}{ Especificação P\&S } \\
\hline & $\begin{array}{c}\text { Volatilidade da } \\
\text { ER real }\left(\mathrm{S}_{\mathrm{i}, \mathrm{t}}\right)\end{array}$ & $\begin{array}{c}\text { Efeito "third } \\
\text { country" ( }\left(\mathrm{S}_{\mathrm{i}, \mathrm{j}, \mathrm{t}}\right)\end{array}$ & $\begin{array}{c}\text { Volatilidade da } \\
\text { ER real }\left(V_{\mathrm{i}, t}\right)\end{array}$ & $\begin{array}{c}\text { Efeito "third } \\
\text { country" }\left(\mathrm{V} 3_{\mathrm{i}, t}\right)\end{array}$ \\
\hline Agricultura & $-3,22^{* \star}$ & $12,74^{*}$ & $-0,59^{* \star}$ & - \\
\hline Pecuária & - & - & - & - \\
\hline Químicos & $-5,81^{*}$ & - & $-1,09^{*}$ & $-1,32^{*}$ \\
\hline Manufaturados & $-7,74^{*}$ & - & $-0,56^{*}$ & $-1,44^{*}$ \\
\hline Mineração e óleo & $-7,79^{*}$ & - & $-0,89^{*}$ & $-1,36^{*}$ \\
\hline Total (todos os setores) & $-5,88^{*}$ & $-2,40^{*}$ & $-0,65^{*}$ & $-0,99^{*}$ \\
\hline
\end{tabular}

$\left(^{*}\right)$ Estatisticamente significativo ao nível de 1\%; $\left(^{*}\right)$ Estatisticamente significativo ao nível de 5\%.

Os resultados para o Mercosul mostram que o uso de duas medidas diferentes de volatilidade da ER real podem produzir resultados similares, tanto em termos de sinais como em interpretação das estimações econométricas do modelo gravitacional. No entanto, os resultados se mostraram ambíguos quando consideramos o efeito "third country".

O uso da medida MSD de volatilidade da ER real produziu não somente estimativas muito diferentes para os coeficientes das variáveis volatilidade da ER real e efeito "third country" em comparação às estimativas obtidas pela especificação P\&S dentro de cada setor, mas também os resultados foram bastante contrastantes entre setores. As diferenças em magnitude entre os coeficientes estimados em ambas especificações já eram esperadas devido às diferenças e particularidades de construção das medidas de volatilidade adotadas neste estudo.

\section{CONCLUSÕES E IMPLICAÇÕES}

Brasil e seus parceiros do Mercosul têm negociado o destino dos acordos para implementação das tarifas externas comuns até 2006, além de maneiras de aumentar o fluxo de comércio através de ações multilaterais duráveis e estáveis, vindo a favorecer uma área de livre comércio mais integrada. As recentes crises econômicas trouxeram 
novos obstáculos às políticas comerciais deste bloco. A falta de coordenação das políticas macroeconômicas adotadas, principalmente por Argentina e Brasil, tem sido uma ameaça ao futuro deste bloco econômico.

Os principais resultados deste estudo indicam que o comércio brasileiro dentro do Mercosul é negativamente afetado não só pelos próprios movimentos cambiais, mas também pela volatilidade cambial dos seus parceiros comerciais dentro do Mercosul. Os impactos da volatilidade da taxa de câmbio variam entre setores, existindo pouca evidência das razões para tais respostas, principalmente se o argumento usado se baseia no modelo de Baldwin e Krugman ("hysteresis model"), o qual prevê que os setores com maiores custos de implementação seriam menos sensíveis à volatilidade da taxa de câmbio (ER) real. O setor de mineração e óleo, juntamente com o setor de manufaturados, se mostraram os mais sensíveis à volatilidade da ER real que, segundo o modelo de Baldwin e Krugman, deveriam indicar que estes setores apresentam as menores exigências de investimento para sua implementação, o que não condiz com a realidade.

A volatilidade da taxa de câmbio de outros países do Mercosul (efeito "third country") mostrou afetar o comércio brasileiro de diferentes formas, de acordo com o setor considerado. Segundo a especificação MSD, como medida de volatilidade cambial, o efeito "third country" foi significativo estatisticamente apenas para o setor agrícola, e para todos os setores considerados conjuntamente. Esta variável mostrou ter uma influência surpreendentemente positiva e de grande magnitude no comércio agrícola, sugerindo que a variabilidade cambial em outros parceiros comerciais contribui para um maior comércio brasileiro no Mercosul. De acordo com a especificação P\&S, o efeito "third country" mostrou-se mais estável entre os diferentes setores, mas nos setores agrícola e pecuário, esta variável não mostrou apresentar efeito significativo. Em outros setores, o efeito "third country" foi negativo e variou de -0,99 a -1,44.

Os principais resultados obtidos pelo presente estudo sugerem que o fluxo de comércio brasileiro no Mercosul é direta e significativamente afetado pela volatilidade da taxa de câmbio entre Brasil e seus parceiros comerciais, pelo papel desempenhado pelas tarifas comerciais e, também, pelos níveis de renda dos países deste bloco econômico. Como esperado, o crescimento da renda e a redução das tarifas comerciais contribuem para aumentar o comércio bilateral no Mercosul. O simples fato de os países-membros do Mercosul, principalmente Argentina e Brasil, terem adotado políticas macroeconômicas tão diferentes e divergentes em anos recentes, podem ter contribuído para um maior impacto da volatilidade da taxa de câmbio real dos países-membros do Mercosul no comércio brasileiro neste bloco. Os resultados sugerem que estas políticas desencontradas podem ser uma das causas da considerável variabilidade cambial e de preços, trazendo impactos adversos ao comércio bilateral devido ao comportamento de aver- 
são ao risco dos agentes econômicos, e devido ao generalizado grau de protecionismo causado e defendido por estes agentes. As principais implicações para políticas que este estudo sugere incluem a implementação de políticas comuns, estáveis e integradas entre os países do Mercosul, de modo a reduzir os impactos adversos da volatilidade cambial no comércio dos países envolvidos. ${ }^{29}$ Como consequiência, regimes cambiais mais estáveis e suaves poderiam ajudar a precaver pressões políticas (lobbying) para aumentar barreiras comerciais quando, por exemplo, a razão de penetração de produtos importados aumenta em determinado país.

Futuras extensões deste estudo devem procurar utilizar dados com maior nível de desagregação, além de outras proxies para a volatilidade da taxa de câmbio, pois alguns resultados obtidos aqui se mostraram ambíguos em termos de sinais e magnitude. A procura por melhor variável instrumental para a medida de volatilidade da taxa cambial, de modo a se testar a presença do viés de simultaneidade, também deve ser incluída em estudos futuros.

\section{REFERENCIAS}

Averbug, A. Mercosul: conjuntura e perspectivas. Revista do BNDES, dezembro de 1998.

Baer, W.; Silva, P.; Cavalcanti, T. Economic Integration without Policy Coordination: the case of Mercosur. Manuscript. University of Illinois, Champaign-Urbana, 2001.

Baldwin, R.E. Hysteresis in import prices: the beachhead effect. The American Economic Review 78, p.773-785, 1988.

; Krugman, P. Persistent trade effects of large exchange rate shocks. Quarterly Journal of Economics 104, p.635-655, 1989.

29 É importante notar que uma maior integração das políticas macroeconômicas no Mercosul se justificaria se este bloco estivesse caminhando para uma área de integração monetária, como aconteceu com a União Européia. Existem vários pré-requisitos a serem atendidos pelos países do Mercosul de modo a seguir esta trajetória, tais como a redução da variabilidade da taxa de câmbio, independência dos bancos centrais, maior flexibilidade de preços e salários, fortalecimento do setor financeiro, dentre outros. Como esta discussão foge do escopo deste artigo, para maiores detalhes ver Eichengreen (1998). O presente estudo busca investigar o impacto de apenas um destes pré-requisitos no comércio brasileiro do Mercosul. Se tal volatilidade cambial é reduzida, vimos que o comércio brasileiro tende a se expandir neste bloco, contribuindo para que a participação do comércio brasileiro do Mercosul aumente em termos relativos, ganhando maior importância em termos de balança comercial. Salientam-se, no entanto, as dificuldades da harmonização de tais políticas, dadas as peculiariedades de cada país. Um exemplo disso são as atuais políticas adotadas por Argentina e Brasil. Se na atual conjuntura o governo argentino prefere adotar uma taxa de câmbio desvalorizada, combinando controle de capitais, imposto sobre exportação, expansão monetária e uma política fiscal frouxa, o modelo brasileiro tem sido exatamente o oposto, mas sem nenhuma tentativa de controle de capitais ou de câmbio. 
Batten, D.S.; M.T. Belongia, M.T. Monetary policy, real exchange rates and U.S. agricultural exports. American Journal of Agricultural Economics 68, p. 422427,1986 .

Bergstrand, J.H. The generalized gravity equation, monopolistic competition, and the factor-proportions theory in international trade. Review of Economics and Statistics, 71(1), p.143-153, 1989.

Bessler, D.A.; Babula, R.A.. Forecasting wheat exports: Do exchange rates matter?. Journal of Business, Economics and Statistics 5, p.397- 406, 1987.

Bittencourt, M.V.L. The impacts of trade liberalization and macroeconomic instability on the Brazilian economy. (Ph.D. Dissertation). The Ohio State University, 2004.

Brodsky, D. Fixed versus flexible exchange rates, and the measurement of exchange rate instability. Journal of International Economics 16, p. 295-306, 1984.

Cho, G. D. Real exchange rate movements and agricultural trade. (Ph.D. Dissertation). The Ohio State University, 2001.

Cho, G., Sheldon, I. M.; McCorriston, S. Exchange rate uncertainty and agricultural trade. American Journal of Agricultural Economics 84 (4), p.932-942, 2002.

Cushman, D.O. US bilateral trade flows and exchange risk during the floating period. Journal of International Economy 24, p.317-330, 1988.

Deardorff, A. Determinants of bilateral trade: does gravity work in a Neoclassical world?. In: Frankel, J. (Ed.). In the Regionalization of the World Economy. Chicago: University of Chicago Press, 1998.

De Grauwe, P. Exchange rate variability and the slowdown in growth of international trade. IMF Staff Papers n.24, 317-330, 1988.

; De Bellefroid, B. Long-run exchange rate variability and international trade. In: S. Arndt, S.; Richardson, J.D. (Eds). Real-Financial Linkages Among Open Economies. Cambridge: MIT Press, 1986.

Dell'Ariccia, G. Exchange rate fluctuations and trade flows. IMF Staff Papers n. 46, p. 293-314, 1999.

Dornbusch, R. Exchange rates and prices. The American Economic Review 77, p.93106, 1987.

Egger, P. An econometric view on the estimation of gravity models and the calculation of trade potentials. The World Economy 25(2), p.297-312, 2002.

Eichengreen, B. Does Mercosur need a single currency? NBER Working Paper n.6821, Cambridge, MA. NBER, 1998.

; Irwin, D.A. Trade blocs and the reorientation of trade in the 1930s. Journal of International Economy 38, p.1-24, 1995.

Evenett, S.J.; Keller, W. On theories explaining the success of the gravity equation. Journal of Political Economy 110 (2), p.281-316, 2002. 
Frankel, J.; Wei, S. Trade blocs and currency blocs. NBER Working Paper n.4335, Cambridge, MA. NBER, 1993.

; Stein, E.; Wei, S. Continental trading blocs: are they natural or supernatural? In: Frankel, J. (Ed.). In the Regionalization of the World Economy. Chicago: University of Chicago Press, 1998.

Froot, K. A. ; Klemperer, P.D. Exchange rate pass-through when market share matters. The American Economic Review 79, p.637-654, 1989.

Haley, S.L.; Krissoff, B. U.S. grain exports and the value of the U.S. dollar. Journal of Agricultural Economics Research 39, p.12-21, 1987.

Helpman, E. Imperfect competiton and international trade: evidence from fourteen industrial countries. Journal of the Japanese and International Economies 1, p.6281, 1987.

Hooper, P.; Kohlhagen, S.W. The effect of exchange rate uncertainty on the prices and volume of international trade. Journal of International Economy 8, p.483$511,1978$.

Kenen, P.; Rodrik, D. Measuring and analysing the effects of short-term volatility in real exchange rates. Review of Economics and Statistics 68, 1986.

Klein, M.W. Sectoral effects of exchange rate volatility on United States exports. Journal of International Money and Finance 9, p.299-308, 1990.

Lanyi, A.; Suss, E. Exchange rate variability: alternative measures and interpretation. IMF Staff Papers n. 29, p.527-560, 1982.

Linnemann, H. An econometric study of international trade flows. Amsterdam: NorthHolland, 1966.

Mark, N. Exchange rates and fundamentals: evidence on long-horizon predictability. The American Economic Review 85, p. 201-218, 1995.

Maskus, K. Exchange rate risk and U.S. trade: a sectoral analysis. Federal Reserve Bank of Kansas Economic Review 3, p.16-28, 1986.

Peree, E.; Steinherr, A.. Exchange rate uncertainty and foreign trade. European Economic Review 33, p.1241-1264, 1989.

Pick, D.H. Exchange rate risk and U.S. agricultural trade flows. American Journal of Agricultural Economics 72, p.694-700, 1990.

; Vollrath, T.L. Real exchange rate misalignment and agricultural export performance in developing countries. Economic Development and Cultural Change 42, p.555-571, 1994.

Rose, A. One money, one market: the effect of common currencies on trade. Economic Policy 30, p.7-46, 2000.

Sauer, C.; A. K. Bohara, A.K. Exchange rate volatility and exports: regional differences between developing and industrialized countries. Review of International Economics 9 (1), p.133-152, 2001. 
Schuh, G. E. The exchange rate and U.S. agriculture. American Journal of Agricultural Economics 56, p.1-13, 1974.

Soloaga, I.; Winters, A. Regionalism in the nineties: What effect on trade? The North American Journal of Economics and Finance 12, p.1-29, 2001.

Thursby, J.G.; Thursby, M.C. Bilateral trade flows, the Linder hypothesis and exchange rate risk. Review of Economics and Statistics 69, p.488-495, 1987.

Tinbergen, J. Shaping the world economy. Twenty-Century Fund, New York, 1962.

Trefler, D. Trade liberalization and the theory of endogenous protection: an econometric study of U.S. import policy. Journal of Political Economy 101 (1), p.138160, 1993.

Tweeten, L. Farm policy analysis. Boulder, CO: Westview Press, 1989.

Wei, S.J. Intra-national versus international trade: How stubborn are nations in global integration? NBER Working Paper n.5531, Cambridge, MA. NBER, 1996. 